\title{
A fluorescence-based in vitro assay for investigating early endosome dynamics
}

\author{
Sina V Barysch ${ }^{1,2}$, Reinhard Jahn ${ }^{1}$ \& Silvio O Rizzoli ${ }^{2}$
}

\begin{abstract}
${ }^{1}$ Department of Neurobiology, Max Planck Institute for Biophysical Chemistry, Göttingen, Germany. ${ }^{2}$ STED Microscopy of Synaptic Function, European Neuroscience Institute, Göttingen, Germany. Correspondence should be addressed to R.J. (rjahn@gwdg.de).

Published online 27 May 2010; doi:10.1038/nprot.2010.84

Early endosomes receive material from the plasma membrane by fusion with endocytotic vesicles. This material is sorted within endosomes and directed to subdomains at which carrier vesicles bud. These vesicles are then transported toward different cellular destinations. In this article, we describe a protocol for the cell-free reconstitution of endosome docking/fusion and sorting/ budding, which is based on labeling of endosomes by endocytotic uptake with fluorescent cargoes. The protocol includes ( $i$ ) the preparation of fluorescently labeled endosomes, (ii) assays for docking/fusion and for sorting/budding in vitro and (iii) imaging of the reaction mix by fluorescence microscopy to quantify docking, fusion, cargo sorting and budding using counting of single organelles. Production of endosome stocks requires approximately $1 \mathrm{~d}$. The in vitro reactions can then be performed separately ( $\sim \mathrm{d})$ and are conveniently carried out with multiple samples in parallel. The assay can be adapted for studying the dynamics of organelles other than endosomes.
\end{abstract}

\section{INTRODUCTION}

Early endosomes represent the first sorting station of newly internalized material in all eukaryotic cells. On endocytosis, material is taken up into small carrier vesicles that are targeted to early endosomes, to which they dock and fuse. Endocytosed material is then sorted within the endosome. Depending on the final destination, such cargoes are either packed into budding vesicles, or they remain within early endosomes until they mature into late endosomes and then fuse with lysosomes for degradation of their content. Carrier vesicles budding from early endosomes are delivered to different intracellular destinations such as the plasma membrane, the recycling endosomes or the trans-Golgi network. Thus, early endosomes undergo continuous remodeling due to a steady influx of material by fusion with incoming endocytotic vesicles, which is balanced by an efflux of outgoing vesicles. Moreover, early endosomes frequently fuse with each other (termed homotypic fusion).

Of all these processes, fusion is the only one to be extensively studied. The reason is that fusion can be followed in vitro by biochemical assays relying on contents mixing. In these assays, two sets of endosomes are labeled separately by endocytosis with two markers that possess an intrinsic affinity toward each other (such as antigen and antibody, or avidin and biotin). After cell lysis, endosomes are allowed to fuse in vitro in the presence of an externally added quencher that prevents complex formation between markers escaping from the organelles. The amount of fusion is determined by measuring the degree of binding between markers (reviewed in ref. 1). Such assays have defined our current knowledge of fusion dynamics, and most of the proteins involved in homotypic fusion were identified in such in vitro fusion experiments. However, these assays cannot address the vital step that precedes fusion-docking. Furthermore, measuring sorting and budding in vitro has been much more difficult, with most assays relying on the separation of small carrier vesicles from larger donor organelles. For example, permeabilized cells were used to measure endosomal budding of recycling vesicles. In contrast to the endosomal donor organelle, these vesicles can escape from cells though pores in the plasma membrane $e^{2,3}$. Moreover, separation through density gradients has been used to study the budding of several types of vesicles from endosome precursors ${ }^{4-8}$. However, these assays only function when there is a substantial difference in buoyancy between the precursor and budded organelles-which explains the paucity of budding assays in the literature.

We have recently developed a new technique for measuring docking and fusion, which is based on the separate labeling of two sets of endosomes with two fluorescent cargo molecules. In vitro fusion of organelles results in mixing of the dyes, producing double-labeled organelles that can be conveniently quantified by fluorescence microscopy ${ }^{9}$. Furthermore, we found that measuring the distance between the centers of intensity of the differentially labeled organelles (see Experimental design) allows for monitoring fusion and docking in paralle ${ }^{10,11}$. Sorting and budding can be investigated by a variation of the technique: organelles are simultaneously labeled with multiple, differently colored markers, and the segregation of markers is followed by monitoring the production of single-color carrier vesicles ${ }^{12}$ (see Experimental design).

Our assay has several fundamental advantages over the assays discussed above. First, to the best of our knowledge, our in vitro assay is the only one to date that is able to measure the docking of small organelles quantitatively. Second, it can analyze (and differentiate) docking and fusion in parallel. Third, our budding and fusion assays have no inherent buoyancy requirements, and therefore can be used for investigating virtually any type of organelles (we have already used them for endosomes from various cell types and even synaptic vesicles $\left.{ }^{13}\right)$. Finally, compared with the biochemical in vitro fusion and budding assays, our technique has the advantage of analyzing cargo-containing endosomes on a single-organelle basis. This has allowed, for example, the investigation of protein composition on single sorting organelles, by immunostaining ${ }^{12}$. From a technical point of view, the assay can be conveniently upscaled, with up to 20 reactions being run in parallel, in a much easier manner than upscaling, for example, density-gradient assays.

The reliance of the assay on identification of different fluorescently labeled cargoes is an advantage, as indicated above; however, it also introduces several constraints. The fusion of two differently colored organelles is accurately reported, but the fusion of two 
Figure 1 | Experimental design of the in vitro endosome docking/fusion and sorting/budding assay. For docking/fusion (left), two sets of cells are allowed to take up different fluorescent cargoes (dextran-Alexa 488 and dextran-Alexa 594) by endocytosis for $5 \mathrm{~min}$, leading to the fluorescent labeling of early endosomes with two colors. Cells are cracked and postnuclear supernatants (PNSs) are prepared, which contain fluorescently labeled endosomes. In vitro incubation of the two PNS fractions in the presence of cytosol and an ATPregenerating system leads to docking and fusion of organelles, which can be visualized by immobilizing them on glass coverslips and subsequent imaging (in which double-labeled organelles appear). Data analysis shows the precise position of each endosome and measures distances between them, thus allowing the quantification of docked and fused endosomes. For sorting/budding (right), cells are simultaneously labeled with two fluorescent cargoes and PNS is prepared. In vitro incubation of the PNS fraction in the presence of cytosol and an ATP-regenerating system leads to cargo sorting and separation because of budding, resulting in a decrease in double-labeled organelles. As for the docking/fusion assay, this can be visualized by fluorescence microscopy and quantified by image analysis.

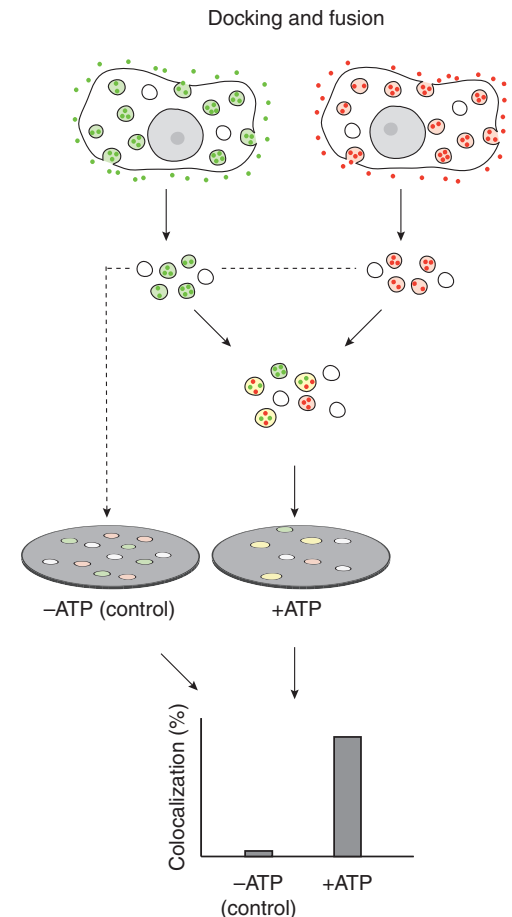

identically colored organelles is not. In addition, only one doublelabeled spot appears when several organelles of different colors fuse together, with the assay thus ignoring multiple fusion events. For the sorting reaction, formation of small vesicles containing both of the fluorescently labeled cargoes is not detected, as they are still double labeled.

\section{Experimental design}

The workflow of the in vitro early endosome docking/fusion or sorting/budding assay is depicted in Figure 1. For these assays, cytosol (here we describe the preparation of cytosol from adult rat brains, Steps 1-8) and fluorescently labeled endosomes are needed (we label early endosomes by incubating cultured cells with externally added fluorescent cargo molecules by endocytotic uptake, Steps 9-24). After labeling, cells are cracked with a ball homogenizer and a postnuclear supernatant (PNS), which contains the labeled endosomes, is prepared (Steps 25-30). For docking/fusion assays, two separate sets of differently labeled endosomes are needed. For sorting/budding assays, endosomes are simultaneously labeled with at least two endocytic markers that are sorted into different vesicular pathways. As our laboratory focuses on neuronal membrane trafficking, we use for our assays the neuroendocrine cell line PC12; however, the assay can be adapted to other cell lines.

In vitro incubation of a reaction mix that contains labeled endosomes, cytosol and an ATP-regenerating system at physiological temperature leads to both docking and fusion, or sorting and budding of the endosomes (Steps 31-39). Depending on the combination of labeled endosomes used, either docking/fusion or sorting/budding is preferentially visualized. The purpose of the assay is to test the function of different molecules in the docking/ fusion or sorting/budding processes. Therefore, inhibitors or activators of different molecules should be tested (see Step 37). Various components can be used, from ion chelators (such as calciumor magnesium-chelating compounds), chemical inhibitors for different processes (such as actin depolymerizing reagents), antibodies for different receptors or effectors (such as clathrin antibodies) to purified effector proteins (e.g., Rab proteins).

To quantify docking/fusion or sorting/budding, we centrifuged the endosomes onto glass coverslips in which they adsorb and can be imaged by epifluorescence microscopy in different color channels (Steps 40-43). Alternatively, it is possible to attach endosomes to coverslips using other approaches such as dilution in low-melt agarose, which is then allowed to harden on the coverslips. For the docking/fusion assay, double-labeled ('yellow') endosomes are visible after incubation. In contrast, sorting/budding leads to the separation of the two labels, resulting in a decrease of doublelabeled endosomes.

To distinguish truly double-labeled (fused) from closely apposed (docked) endosomes, we use the following data analysis procedure (Steps 44-53): for each labeled particle a center of intensity is determined in the respective color channel, thus providing information about its precise localization. The distance between the closest neighboring spots (endosomes) in different colors serves as a readout for docking, fusion, sorting and budding. Note that the ability to determine the distance between the two organelles in two channels (i.e., the ability to determine the precise position of each organelle in its respective color channel) is fully independent of diffraction, unlike the situation in which both organelles are similarly labeled. Therefore, this procedure allows information to be obtained beyond the diffraction limit. To correct for drifts between the images acquired in the different channels, we use multicolored fluorescent beads for alignment. The analysis can be performed using various image analysis programs such as Metamorph (Universal Imaging Corporation), ImageJ (NIH), Matlab (The Mathworks Inc.) or Excel (Microsoft Corporation).

As an important control, the procedure should also be performed in conditions in which only multicolor fluorescent beads are added. This allows the determination of the minimal distance between the centers of intensity of perfectly colocalizing objects-a parameter that may vary between different microscopy setups (see Step 50). 


\section{MATERIALS}

\section{REAGENTS}

- Adult Wistar rats (! CAUTION Experiments involving animals must be carried out in accordance with all appropriate national and institutional regulations.)

- PC12 cells (clone 251), a similar PC12 line can be obtained commercially from the American Type Culture Collection (ATCC, cat. no. CRL-1721.1)

- Dulbecco's modified Eagles's medium (DMEM; Lonza, cat. no. 12-733F)

- Glutamine (Lonza, cat. no. 17-605E)

- Penicillin-streptomycin (Lonza, cat. no. 17-602E)

- Fetal calf serum (FCS Gold; PAA Laboratories, cat. no. A15-649)

- Horse serum (Biochrom, cat. no. S9135)

- Trypsin/EDTA (Lonza, cat. no. 17-161E)

- TetraSpeck microspheres, $0.2 \mu \mathrm{m}$ (Invitrogen, cat. no. T7280)

- Fluorescent cargo (all Invitrogen): transferrin-Alexa 488 (T13342), LDL-DiI (L3482), cholera toxin subunit B-Alexa 647 (C34778), dextran-Alexa 488 (D22910) and dextran-Alexa 594 (D22913)

- Protease inhibitors: leupeptin hemisulfate (AppliChem, cat. no. A2183), aprotinin (AppliChem, cat. no. A2132), pepstatin A (Peptide Institute, cat. no. 4039), PMSF (Roth, cat. no. 6367.3), alternatively, Complete EDTA-Free protease inhibitor tablets (Roche, cat. no. 1873580)

- OptiMEM (Invitrogen, cat. no. 31985)

- Creatine phosphate (Roche, cat. no. 621722)

- Creatine kinase (Roche, cat. no. 127566)

- ATP (AppliChem, cat. no. A1348)

- HEPES (Gerbu Biotechnik, cat. no. 1009)

- Magnesium acetate (Merck, cat. no. 1.05819)

-1,4-DTT (Roth, cat. no. 6908.2)

- Potassium acetate (Merck, cat. no. 1.04936)

- Hexokinase (Roche, cat. no. 1426362)

- Sodium chloride, $\mathrm{NaCl}$ (Merck, cat. no. 1.06404)

- Disodium hydrogen phosphate, $\mathrm{Na}_{2} \mathrm{HPO}_{4}$ (Merck, cat. no. 1.06580)

- Sucrose (Merck, cat. no. 1.07687)

- Imidazole (AppliChem, cat. no. A10730)

- Bovine serum albumin, BSA (AppliChem, cat. no. A1391)

- D-Glucose (Merck, cat. no. 1.08342)

- Ethanol (Merck, cat. no. 1.00983)

- Sodium hydroxide, $\mathrm{NaOH}$ (Merck, cat. no. 1.06498)

- Potassium hydroxide, $\mathrm{KOH}$ (Merck, cat. no. 1.05033)

- Hydrochloric acid, $\mathrm{HCl}$ (Merck, cat. no. 1.09057)

EQUIPMENT

- Dissection tools

- Standard cell culture equipment (laminar flow hoods, incubators, cooling centrifuges, water baths, refrigerator and freezers, cell culture plates and flasks)

- Plastic flasks (15-ml and 50-ml tubes and flasks of different sizes) and plastic pipettes $(5,10,25$ and $50 \mathrm{ml}) \boldsymbol{\Delta}$ CRITICAL All steps must be performed in the absence of detergent traces, i.e., using plasticware or using specially cleaned glassware.

- Centrifuge, Sorvall RC5B Plus, with a SS-34 rotor and polycarbonate flanged tubes (Sorvall, cat. no. 03146) $\Delta$ CRITICAL Tubes must be free of detergent traces.

- Ultracentrifuge, Beckman TL-100, with a TLA-100.3 rotor and polycarbonate tubes (Beckman, cat. no. 349622) $\boldsymbol{\Delta}$ CRITICAL Tubes must be free of detergent traces.

- Glass/Teflon Potter Elvehjem Tissue Grinder, 55 ml (OMNI International, cat. no. 07-358054)

- Overhead stirrer (Kika Labortechnik, cat. no. RW20 DZM.n)

- Ball homogenizer (Isobiotech, cat. no. 20209 547.9, see EQUIPMENT SETUP)

- Disposable syringe, NORM-JECT (1 ml Ins/TBC; Henke-Sass \& Wolf)

$\triangle$ CRITICAL This should be free of latex and silicon oils.

- Refrigerated table-top centrifuge (Eppendorf, cat. no. $5415 \mathrm{R}$ )

- Reaction tubes, polycarbonate, $0.2 \mathrm{ml}$ (Beckman, cat. no. 343775)

- Lid for reaction tubes: rubber sealing from 1-ml syringes (Dispomed, cat. no. 22009)

- 12-well plates (Corning Costar, 3513)

- 18-mm glass coverslips (Marienfeld Superior, cat. no. 0111580)

- Plate centrifuge (Heraeus Multifuge $4 \mathrm{KR}$ ) with a rotor and inlays for plates (Heraeus HIGHplate windshielded rotors)
- Open imaging chamber for 18-mm coverslips (see EQUIPMENT SETUP)

- Epifluorescence microscopy setup (see EQUIPMENT SETUP)

- Bath sonicator (Bandelin Sonorex RK 100)

- Dark (covered) water bath with an agitation device (GFL 1086)

- Forceps

- Pasteur pipettes (plastic)

- Zeiss Axiovert 200M fluorescence microscope (see EQUIPMENT SETUP)

- METAMORPH (Universal Imaging Corporation)

- Matlab (The Mathworks Inc.)

REAGENT SETUP

$\Delta$ CRITICAL All solutions and buffers must be free of detergent and therefore should be prepared in plasticware, or in glassware thoroughly cleaned with ethanol. All reagents that can be stored in aliquots at $-20^{\circ} \mathrm{C}$ can be used for at least 6 months after preparation.

PC12 culture medium The medium consists of DMEM supplemented with $10 \%(\mathrm{vol} / \mathrm{vol}$ ) horse serum, $5 \%$ (vol $/ \mathrm{vol}$ ) fetal calf serum, $4 \mathrm{mM}$ glutamine and $100 \mathrm{U} \mathrm{ml}^{-1}$ each of penicillin and streptomycin. It can be stored at $4{ }^{\circ} \mathrm{C}$ and used within 1-2 weeks after preparation.

Sucrose buffer The solution consists of $320 \mathrm{mM}$ sucrose and $5 \mathrm{mM}$ HEPES, $\mathrm{pH} 7.4$ (adjusted with $\mathrm{NaOH}$ ). It can be stored at $4{ }^{\circ} \mathrm{C}$ and used within 1 week after preparation.

Homogenization buffer The solution consists of $250 \mathrm{mM}$ sucrose and $3 \mathrm{mM}$ imidazole, $\mathrm{pH} 7.4$ (with $\mathrm{HCl}$ ). It can be stored at $4{ }^{\circ} \mathrm{C}$ and used within 1 week after preparation.

PMSF stock solution The solution consists of $200 \mathrm{mM}$ PMSF in ethanol. It can be stored at $-20^{\circ} \mathrm{C}$ and used within 1 week after preparation.

Pepstatin A stock solution The solution consists of $1 \mathrm{mg} \mathrm{ml}^{-1}$ pepstatin A in DMSO. It can be stored in aliquots at $-20^{\circ} \mathrm{C}$.

Leupeptin stock solution It comprises $10 \mathrm{mg} \mathrm{ml}^{-1}$ leupeptin hemisulfate in $\mathrm{H}_{2} \mathrm{O}$. It can be stored in aliquots at $-20^{\circ} \mathrm{C}$.

Aprotinin stock solution The solution is prepared by diluting $10 \mathrm{mg} \mathrm{ml}^{-1}$ aprotinin in $\mathrm{H}_{2} \mathrm{O}$ and can be stored in aliquots at $-20^{\circ} \mathrm{C}$.

PBS The solution contains $150-\mathrm{mM} \mathrm{NaCl}$ and $20-\mathrm{mM} \mathrm{Na}_{2} \mathrm{HPO}_{4}$

(disodium hydrogen phosphate), $\mathrm{pH} 7.4$ (with $\mathrm{HCl}$ ). It can be stored at room temperature (i.e., at $21^{\circ} \mathrm{C}$ ) for up to 2 months.

PBS-BSA It should be prepared freshly for PNS preparation and consist of $5 \mathrm{mg} \mathrm{ml}^{-1}$ BSA in PBS.

DHM buffer The solution consists of $625 \mathrm{mM}$ HEPES, $75 \mathrm{mM}$ magnesium acetate and $10 \mathrm{mM}$ DTT ( $\mathrm{pH} 7.4$, with $\mathrm{KOH})$, and can be stored in aliquots at $-20^{\circ} \mathrm{C}$.

KAc buffer The solution consists of $1 \mathrm{M}$ potassium acetate in $\mathrm{H}_{2} \mathrm{O}$ and can be stored in aliquots at $-20^{\circ} \mathrm{C}$.

ATP solution The solution consists of $100 \mathrm{mM}$ ATP in $\mathrm{H}_{2} \mathrm{O}$ (pH 7.4, with $\mathrm{KOH}$ ) and can be stored in aliquots at $-20^{\circ} \mathrm{C}$.

CP solution It comprises $800 \mathrm{mM}$ creatine phosphate in $\mathrm{H}_{2} \mathrm{O}$ and is stored in aliquots at $-20^{\circ} \mathrm{C}$.

CK solution It comprises $4 \mathrm{mg} \mathrm{ml}^{-1}$ creatine kinase $\left(=3,200 \mathrm{U} \mathrm{ml}^{-1}\right)$ in $\mathrm{H}_{2} \mathrm{O}$ and is stored in aliquots at $-20{ }^{\circ} \mathrm{C}$.

Glucose solution It consists of $250 \mathrm{mM}$ glucose in $\mathrm{H}_{2} \mathrm{O}$ and can be stored in aliquots at $-20^{\circ} \mathrm{C}$.

Internalization medium The internalization medium consists of $1 \mathrm{~g}$ D-glucose in one bottle (500 ml) OptiMEM and is stored in aliquots at $-20^{\circ} \mathrm{C}$. EQUIPMENT SETUP

Epifluorescence microscopy setup The setup consists of a Zeiss Axiovert 200M fluorescence microscope (Carl Zeiss AG) equipped with a $\times 100,1.4$ numerical aperture oil objective and a CCD camera with a 1,317 × 1,035 Kodak chip (pixel size $6.8 \mu \mathrm{m} \times 6.8 \mu \mathrm{m}$; Princeton Instruments Inc.). Blue fluorescence is detected using the excitation filter $350 / 50 \mathrm{D}$, the beamsplitter $400 \mathrm{DCLP}$ and the emission filter 460/50 D. Alexa 488 fluorescence (absorption/emission maxima 496/519 $\mathrm{nm}$ ) is detected with the excitation filter $480 / 40 \mathrm{HQ}$, the beamsplitter $505 \mathrm{LP}$ $\mathrm{Q}$ and the emission filter 527/30 HQ. DiI fluorescence (absorption/emission maxima 554/571) is detected using the excitation filter 545/30 HQ, the beamsplitter $570 \mathrm{LP}$ Q and the emission filter 610/75 HQ. Alexa 594 fluorescence (absorption/emission maxima $\sim 590 / 617 \mathrm{~nm}$ ) is detected using the excitation filter 560/55 HQ, the beamsplitter $595 \mathrm{LP}$ Q and the emission filter 645/75 HQ. Alexa 647 fluorescence (absorption/emission maxima $650 / 665 \mathrm{~nm}$ ) is detected 
using the excitation filter $620 / 60 \mathrm{HQ}$, the beamsplitter $660 \mathrm{LP} \mathrm{Q}$ and the emission filter 700/75 HQ. All filters can be purchased from Chroma. For image acquisition, we use METAMORPH (Universal Imaging Corporation).

Ball homogenizer The homogenizer we use is manufactured by our in-house instrument shop and consists of a stainless steel shaft and a stainless steel ball (Spheric-Trafalgar Ltd). The clearance between the shaft and the ball has to be $20 \mu \mathrm{m}$. The ball homogenizer has been originally described in reference 14 in great detail. A commercially available variant of the ball homogenizer can be obtained from Isobiotech. $\Delta$ CRITICAL Clearance has to be $20 \mu \mathrm{m}$ for PC12 cells and may be different for other cells, e.g., $25 \mu \mathrm{m}$ for BHK cells. Open imaging chamber for 18 -mm coverslips For imaging, we use a chamber that is manufactured by our in-house instrument shop, consisting of a circular metal base on which a plastic ring is screwed, thus holding the coverslip (see ref. 15 for a detailed description). We use this chamber, as it allows imaging in the presence of buffer. Any other design that allows imaging of coverslips is appropriate.

\section{PROCEDURE}

\section{Preparation of rat brain cytosol $\bigcirc$ TIMING 4-5 $\mathrm{h}$}

1| Prepare sucrose buffer with protease inhibitors $\left(1 \mu \mathrm{g} \mathrm{ml}^{-1}\right.$ pepstatin $\mathrm{A}(1: 1,000 \mathrm{vol} / \mathrm{vol}$ of pepstatin A stock solution) and 0.2-mM PMSF (1:1000 vol/vol of PMSF stock solution)) for about $5 \mathrm{ml}$ per rat brain.

$\triangle$ CRITICAL STEP Stir the buffer thoroughly while slowly adding PMSF stock solution, as PMSF might precipitate otherwise.

2| Kill 10-40 rats and remove their brains into a beaker with sucrose buffer (without protease inhibitors, kept on ice). ! CAUTION Follow national and institutional guidelines for animal handling.

3| Wash brains 2-3 times with ice-cold sucrose buffer (without protease inhibitors) to remove most of the blood remaining in the solution.

4| Add 10 brains into the Glass/Teflon Potter Elvehjem Tissue Grinder, add about $5 \mathrm{ml}$ ice-cold sucrose buffer (with protease inhibitors, from Step 1) per brain and homogenize brains by 10 strokes (one stroke consists of one up and down movement) at 900 r.p.m. with an overhead stirrer.

! CAUTION Do not apply too much pressure during the first stroke, as the first homogenization of brains is difficult and should be performed slowly; the glass homogenizer might break otherwise.

5| Centrifuge homogenate at 3,000 (5,000 r.p.m. in a Sorvall SS-34 rotor) for $10 \mathrm{~min}$ at $4^{\circ} \mathrm{C}$. Transfer the supernatant into a fresh tube and discard the pellet.

6| Centrifuge the supernatant at 32,000g (16,500 r.p.m. in a Sorvall SS-34 rotor) for 15 min at $4{ }^{\circ} \mathrm{C}$. Transfer the supernatant into a fresh tube and discard the pellet.

7| Centrifuge the supernatant at minimum 100,000 (we use 330,000g (90,000 r.p.m. in a Beckman 100.3 rotor)) for $30 \mathrm{~min}$ at $4{ }^{\circ} \mathrm{C}$ to obtain the cytosol in the supernatant.

8| Transfer the cytosol-containing supernatant into a fresh tube and divide into small aliquots $(0.3-1 \mathrm{ml})$, determine the protein concentration using a standard assay ${ }^{16}$, snap-freeze the aliquots in liquid nitrogen and store them at $-80{ }^{\circ} \mathrm{C}$. The protein concentration of the cytosol concentrations should be in the range between 6 and $10 \mathrm{mg} \mathrm{ml}^{-1}$.

PAUSE POINT If stored at $-80^{\circ} \mathrm{C}$, rat brain cytosol can be used for at least 12 months.

\section{Culture of PC12 cells TIMING 30 min}

9| Split PC12 cells 1:6 into 6-30 plates $(\emptyset 15 \mathrm{~cm})$ using standard procedures for passaging PC12 cells (or any other cell line) ${ }^{17}$. $\triangle$ CRITICAL STEP Dissociate the cells thoroughly, as otherwise clumps may remain that may lead to inefficient cargo uptake. We resuspend the cell pellet in $5 \mathrm{ml}$ fresh PC12 culture medium and break cell aggregates by passing the cells 20 times though a $200-\mu$ l pipette tip attached to a $10-\mathrm{ml}$ plastic pipette.

10| Allow cells to grow for several days until they reach $80 \%$ confluence.

$\triangle$ CRITICAL STEP Cells should reach a high degree of confluence; otherwise, it is difficult to detach them from the plates.

\section{Preparation of postnuclear supernatants $\bigcirc$ TIMING 3-5 $\mathrm{h}$}

11| Prepare PBS-BSA (freshly, $30 \mathrm{ml}$ for each labeled PNS) and store on ice, together with thawed aliquots of internalization medium (15 ml per PNS), homogenization buffer (50 $\mathrm{ml}$ per PNS) and PBS (10 $\mathrm{ml}$ per PNS).

12 Prewarm trypsin/EDTA and PBS $\left(37^{\circ} \mathrm{C}\right)$, but store PC12 culture medium in the refrigerator.

13| Remove old medium from 6-12 plates of cells and wash each plate with $5 \mathrm{~mL}$ PBS. 
14| Add $2 \mathrm{ml}$ trypsin/EDTA per plate and incubate at room temperature with strong shaking.

$\Delta$ CRITICAL STEP Cells should be exposed to trypsin/EDTA only until they start detaching from the plates. Longer exposure to trypsin/EDTA might cause the degradation of receptors that are required for the uptake of fluorescent cargoes.

15 Stop the reaction with $5 \mathrm{ml}$ cold PC12 medium per plate, collect the cells from the plates (using a 10-ml plastic pipette) and transfer them into $50-\mathrm{ml}$ plastic tubes.

$\triangle$ CRITICAL STEP Cells need to be detached, which may require mechanical force such as repetitively squirting of the medium onto the plate. This procedure is easier when longer trypsin/EDTA incubations have been used (Step 14); it is more efficient in highly confluent cultures (Step 10).

16| Leave tubes on ice and repeat Steps 13-15 for the next 6-12 plates of cells.

17| When all cells are collected, centrifuge all tubes at $250 \mathrm{~g}$ for 5 min at $4{ }^{\circ} \mathrm{C}$.

18| Discard the supernatant and resuspend the cell pellet in $10 \mathrm{~mL}$ of ice-cold PBS and transfer to a 15-ml plastic tube. Centrifuge again at $250 \mathrm{~g}$ for $5 \mathrm{~min}$ at $4^{\circ} \mathrm{C}$.

19| Discard the supernatant and resuspend the cell pellet in $10 \mathrm{ml}$ ice-cold internalization medium and centrifuge the cells as before $\left(250 \mathrm{~g}, 5 \mathrm{~min}, 4^{\circ} \mathrm{C}\right)$. If more than one PNS label is needed (e.g., for docking/fusion assay) and two differently labeled PNS fractions are required, resuspend the cell pellet in $20 \mathrm{ml}$ ice-cold internalization medium and divide into equal amounts in two $15-\mathrm{ml}$ plastic tubes before centrifugation $\left(250 \mathrm{~g}, 5 \mathrm{~min}, 4^{\circ} \mathrm{C}\right)$, for the separate PNSs.

20| Estimate the volume of the resulting cell pellet(s).

$\triangle$ CRITICAL STEP This is just an approximation but is necessary for calculating the amounts of fluorescent cargoes to be added. The volume should be in the range of $0.5-3 \mathrm{ml}$.

21| Remove supernatants and warm the pellets at $37^{\circ} \mathrm{C}$ for 5 min in a water bath.

22 Add fluorescent cargoes in uptake medium to the cell pellets, as described in the options below, and incubate for 5 min at $37^{\circ} \mathrm{C}$. The medium should have the same volume as the estimated cell pellet(s) and should thus show cargo concentrations twice as high as finally desired (e.g., to label $1 \mathrm{ml}$ cell pellet with $50 \mu \mathrm{g} \mathrm{ml}^{-1}$ fluorescent transferrin, $1 \mathrm{ml}$ uptake medium with a concentration of $100 \mu \mathrm{g} \mathrm{ml}^{-1}$ transferrin is required). The cargo composition is dependent on whether the docking/fusion assay (A) or the sorting/budding assay (B) should be used as follows:

(A) Docking/fusion assay

(i) Incubate one set of cells with $500 \mu \mathrm{g} \mathrm{ml}^{-1}$ dextran Alexa 488, $10 \mathrm{kDa}$ (prepared at $1 \mathrm{mg} \mathrm{ml}^{-1}$ in internalization medium).

(ii) Incubate a second set of cells with $500 \mu \mathrm{g} \mathrm{ml}^{-1}$ dextran Alexa 594, $10 \mathrm{kDa}$ (prepared at $1 \mathrm{mg} \mathrm{ml}^{-1}$ in internalization medium).

(B) Sorting/budding assay

(i) For dual labeling with transferrin and LDL: simultaneously incubate cells with $50 \mu \mathrm{g} \mathrm{ml}^{-1}$ transferrin-Alexa 488 and $3 \mu \mathrm{g} \mathrm{m}^{-1} \mathrm{LDL}$-DiI (prepared at $100 \mu \mathrm{g} \mathrm{ml}^{-1}$ transferrin and $6 \mu \mathrm{g} \mathrm{ml}^{-1} \mathrm{LDL}$ in internalization medium).

$\triangle$ CRITICAL STEP LDL easily oxidizes, which affects the uptake efficiency. The exact concentration may need to be adjusted, with concentrations of up to $10 \mu \mathrm{g} \mathrm{ml}^{-1}$ being needed after several weeks of storage. To delay the oxidation process, it is recommended to keep the LDL-DiI stock solution (Invitrogen) always sealed with parafilm.

(ii) For triple labeling with transferrin, $\mathrm{LDL}$ and cholera toxin, incubate cells with $50 \mathrm{\mu g} \mathrm{ml}^{-1}$ transferrin-Alexa 488 , $3 \mu \mathrm{g} \mathrm{ml}^{-1} \mathrm{LDL}$ (both as above) and $3 \mu \mathrm{g} \mathrm{ml}^{-1}$ cholera toxin subunit B-Alexa 647 (i.e., a concentration of $6 \mu \mathrm{g} \mathrm{ml}^{-1}$ for the uptake medium). Note that any other combinations of labeled endocytotic cargoes can be used.

23 Stop the uptake reaction by chilling the cells on ice, centrifuge them at $250 \mathrm{~g}$ for 5 min at $4^{\circ} \mathrm{C}$ and remove the uptake medium.

24| Wash the cell pellet(s) three times with $10 \mathrm{ml}$ ice-cold PBS-BSA; centrifuge after each wash at $250 \mathrm{~g}$ for $5 \mathrm{~min}$ at $4{ }^{\circ} \mathrm{C}$.

25| Prepare 30-50 ml homogenization buffer with protease inhibitors by adding PMSF, pepstatin A, leupeptin and aprotinin $(1: 1,000(\mathrm{vol} / \mathrm{vol})$ of the respective stock solutions). 
26| Wash the cell pellet(s) once with ice-cold homogenization buffer and resuspend the pellet(s) in ice-cold homogenization buffer with protease inhibitors (Step 25).

$\Delta$ CRITICAL STEP Use no more than $4 \times$ the volume of the cell pellet or the PNSs will be too dilute.

27| Crack the cells with a ball homogenizer on ice. Use 1-ml disposable syringes for passing $1 \mathrm{ml}$ of resuspended cells 10-20 times through the precooled ball homogenizer. Repeat the procedure, depending on the volume of cells.

$\Delta$ CRITICAL STEP Before cell cracking, wash the assembled ball homogenizer thoroughly with homogenization buffer (with protease inhibitors, Step 25) to remove air bubbles that would cause damage to the endosomes.

28| Transfer the cell homogenate into 1.5 - $\mathrm{ml}$ tubes and centrifuge at $1,200 \mathrm{~g}$ for $15 \mathrm{~min}$ at $4^{\circ} \mathrm{C}$. This will lead to a separation of the homogenate into two layers: the bottom fraction is the nuclear pellet and the upper fraction is the PNS.

$\triangle$ CRITICAL STEP It is sometimes difficult to see the separation of the two layers properly. Use a bright light source (lamp or bright window) to better illuminate the samples. If no band is visible, further dilute the samples with ice-cold homogenization buffer (with protease inhibitors, Step 25) and repeat the centrifugation process.

29| Remove the supernatant fractions into a new tube and determine the protein concentration of the PNS using a standard method $^{16}$. The PNS concentration is usually between 10 and $16 \mathrm{mg} \mathrm{ml}^{-1}$.

? TROUBLESHOOTING

30| Prepare small aliquots $(100-400 \mu \mathrm{l})$, snap-freeze in liquid nitrogen and store at $-80^{\circ} \mathrm{C}$.

PAUSE POINT If stored at $-80^{\circ} \mathrm{C}$, PNS fractions can be used for at least 12 months.

\section{Preparation of docking/fusion or sorting/budding reactions TIMING 2-4 $\mathrm{h}$}

31| Thaw aliquots of PNS (from Step 30), cytosol (from Step 8), DHM buffer, KAc buffer, ATP solution, CP solution, CK solution and homogenization buffer.

$\triangle$ CRITICAL STEP Be careful to thaw PNS and cytosol slowly on ice to avoid unwanted protein degradation or breaking of organelles. This might require up to $30 \mathrm{~min}$, depending on the size of the aliquots.

$\Delta$ CRITICAL STEP Store aliquots of PNS in the dark to avoid bleaching of the fluorescent cargoes.

32| Sonicate multifluorescent TetraSpeck beads for $2-5 \mathrm{~min}$ in the bath sonicator and prepare a 1:1,000 (vol/vol) bead stock solution in PBS $\left(2-\mu \mathrm{l}\right.$ beads in $2 \mathrm{~mL}$ PBS). This can be kept at $4{ }^{\circ} \mathrm{C}$ for up to 2 weeks.

33| Wash 18-mm glass coverslips in 100\% ethanol and place them into 12-well tissue culture plates.

34| Dilute bead stock solution (from Step 32) 1:100 (vol/vol) in PBS to obtain the final bead solution and pipette $1 \mathrm{ml}$ of it into each well. Remove the air bubbles that might be present between the plate and the coverslip to avoid coverslips floating on centrifugation.

35| Centrifuge plates at 5,880 g for $45 \mathrm{~min}$ at $4^{\circ} \mathrm{C}$ (we use 5,460 r.p.m. in a Heraeus Multifuge); this fixes the beads onto the coverslips. Do not remove the solution after centrifugation.

36| Prepare cytosol mix in the following order on ice, depending on whether an ATP-regenerating system (A) or an ATP-depleting system (B) is required, according to whether a positive control (in presence of ATP) or a negative control (in absence of ATP) is desired:

\section{(A) ATP-regenerating system}

(i) Prepare cytosol mix (volumes correspond to one reaction of $50 \mu \mathrm{l}$, scale up accordingly): $100 \mu \mathrm{g}$ cytosol (usually 10-17 $\mu \mathrm{l}$, depending on the concentration), $0.9 \mu \mathrm{L} \mathrm{DHM}$ buffer, $2.25 \mu \mathrm{l} \mathrm{KAc}$ buffer, $1.67 \mu \mathrm{l}$ ATP solution, $1.67 \mu \mathrm{l} \mathrm{CP}$ solution and $1.67 \mu \mathrm{l} \mathrm{CK}$ solution.

The final concentration of the reaction corresponds therefore to $100 \mu \mathrm{g}$ cytosol, $11.25 \mathrm{mM}$ HEPES, $1.35 \mathrm{mM}$ magnesium acetate, $0.18 \mathrm{mM}$ DTT, $45 \mathrm{mM}$ potassium acetate, $3.3 \mathrm{mM}$ ATP, $26.7 \mathrm{mM}$ creatine phosphate and $6.7 \mu \mathrm{g}$ creatine kinase (=5.3 U).

(B) ATP-depleting system

(i) Centrifuge $5 \mu$ l hexokinase $\left(1,500 \mathrm{U} \mathrm{ml}^{-1}\right.$, supplied as ethanol precipitate) at $16,000 \mathrm{~g}$ for 2 min at $4{ }^{\circ} \mathrm{C}$.

(ii) Remove the supernatant and resuspend the hexokinase pellet in $5 \mu$ glucose solution $(250 \mathrm{mM})$.

(iii) Cytosol mix (volumes correspond to one reaction of $50 \mu \mathrm{l}$; scale up accordingly): $100 \mu \mathrm{g}$ cytosol (usually 10-17 $\mu \mathrm{l}$, depending on the concentration), $0.9 \mu \mathrm{LHM}$ buffer, $2.25 \mu \mathrm{l} \mathrm{KAc}$ buffer and $5 \mu$ l hexokinase-glucose buffer (see above). 
The final concentration of the reaction therefore corresponds to $100 \mu \mathrm{g}$ cytosol, $11.25 \mathrm{mM}$ HEPES, $1.35 \mathrm{mM}$ magnesium acetate, $0.18 \mathrm{mM}$ DTT, $45 \mathrm{mM}$ potassium acetate, $7.5 \mathrm{U}$ hexokinase and $25 \mathrm{mM}$ glucose.

37| Prepare docking/fusion (A) or sorting/budding (B) reactions in $200 \mu$ l polycarbonate centrifuge tubes on ice.

$\Delta$ CRITICAL STEP Pipette quickly to avoid warming of the solutions. Certain processes (e.g., docking or aggregation) might take place even during short pipetting times.

\section{(A) Docking/fusion reaction}

(i) Add cytosol mix (from Step 36, usually between 18 and $25 \mu \mathrm{l}$ ).

(ii) Add chemicals, inhibitory/modulating proteins, peptides, antibodies (optional; see Experimental design for further information).

(iii) Add $100 \mu$ g PNS containing dextran-Alexa 488 and $100 \mu$ g PNS containing dextran-Alexa 594 (usually between 5 and $8 \mu$ l each).

(iv) Adjust to $50 \mu \mathrm{l}$ with homogenization buffer.

\section{(B) Sorting/budding reaction}

(i) Add cytosol mix (from Step 36, usually between 18 and $25 \mu \mathrm{l}$ ).

(ii) Add chemicals, inhibitory/modulating proteins, peptides, antibodies (optional; see Experimental design for further information).

(iii) Add $200 \mu \mathrm{g}$ PNS containing transferrin-Alexa 488 and LDL-DiI (or other double- or triple-labeled PNS) (usually between 10 and $15 \mu \mathrm{l})$.

(iv) Adjust to $50 \mu$ with homogenization buffer.

38| Close reaction tubes with a lid and incubate reactions for $45 \mathrm{~min}$ at $37^{\circ} \mathrm{C}$ with a slow agitation (speed: 20 shakes per minute in the GFL 1086 water bath). Leave controls on ice for $45 \mathrm{~min}$.

$\Delta$ CRITICAL STEP Samples should be kept in the dark to avoid bleaching the fluorescent dyes.

39| Place the samples on ice for some minutes to cool, pipette $7 \mu \mathrm{l}$ of each reaction onto the bead-containing solution on the coverslips (from Step 35) and centrifuge the 12-well plates at 5,880g (5,460 r.p.m. in a Heraeus Multifuge) for 45 min at $4^{\circ} \mathrm{C}$.

\section{Imaging of the samples TIMING 0.5-6 $\mathrm{h}$}

40| Place one coverslip into the open imaging chamber for 18-mm coverslips, cover it with PBS and focus the samples.

41| Acquire images in the channels of the respective label (Alexa 488, DiI, Alexa 594, Alexa 647, depending on the PNS fractions that are used) and in one additional channel in which only fluorescent beads can be visualized (e.g., blue) with an epifluorescence microscope (see EQUIPMENT SETUP).

$\triangle$ CRITICAL STEP Be careful with bleedthrough between the different channels and adjust the exposure times to minimize it.

\section{? TROUBLESHOOTING}

42| Acquire images from 6 to 20 random areas for each coverslip.

43| Repeat Steps 40-42 with the remaining coverslips.

PAUSE POINT After saving the images, data analysis can be performed at any time.

\section{Data analysis $\bigcirc$ TIMING up to several hours}

44| Filter the images with an unsharp filter, which sharpens the images by subtracting a blurred (unsharp) version of the image from itself, and eliminates variations across an uneven field (for more information see ref. 18). Different blurring procedures can be used; we found the imopen function in Matlab (The Mathworks Inc.) to be optimal.

45 Threshold the images so that only those spots that represent fluorescent objects (beads and endosomes) remain. $\triangle$ CRITICAL STEP Using too high thresholds will result in the identification of too few spots (only the brightest ones), whereas using too low thresholds will lead to the identification of background spots in addition to the real ones. Both cases are critical, as they will badly distort the analysis.

? TROUBLESHOOTING 
46| Find the spots in the blue images (the images in which the beads are the only fluorescent objects) and calculate the centers of intensity in the $x$ and $y$ axes:

$$
x_{i}=\frac{\sum_{j=1}^{N} x_{j} I_{j}}{\sum_{j=1}^{N} I_{j}} \quad y_{i}=\frac{\sum_{j=1}^{N} y_{j} I_{j}}{\sum_{j=1}^{N} I_{j}}
$$

where $N$ is the number of pixels in the spot, $x$ and $y$ the coordinates of each pixel in the spot and $I$ the intensity of each pixel in the spot.

47| Calculate the intensity centers of all spots in the other channels (e.g., green and red).

48 Identify the intensity centers of the beads in the endosome color channels (they represent the spots in the green/red channels that are closest to the blue bead spots). We will refer to the bead coordinates in green and red as $\mathrm{GB}_{x^{\prime}} \mathrm{GB}_{y}$ and $\mathrm{RB}_{x^{\prime}}$ $\mathrm{RB}_{\mathrm{y}^{\prime}}$, respectively (where B stands for 'bead', $\mathrm{G}$ for 'green' and $\mathrm{R}$ for 'red'). Thereafter, correct the shift in $x$ and $y$ directions between the color channels for all endosomes, bearing in mind that any shift between the green and red coordinates of the beads applies to all other spots in the images. The shift in the $x$ direction is $\mathrm{RB}_{x}-\mathrm{GB}_{x^{\prime}}$ the shift in the $y$ direction is $\mathrm{RB}_{y}-\mathrm{GB}_{y^{*}}$ To correct for the shift, it is recommended to apply the following formulas for each set of endosome coordinates in the red channel (referred to as $R E_{x}$ and $R E_{y^{\prime}}$ where $E$ stands for 'endosome'): $R E_{x}=R E_{x}-\left(R B_{x}-G B_{x}\right)$ and $R E_{y}=R E_{y}-\left(R B_{y}-G_{y}\right)$.

49| For every endosome (E) in one channel, calculate the distance $(d)$ to its closest neighbor in the other channel. This is performed by calculating the distances to all endosomes in the other channel, using the Pythagorean formula:

$$
d=\sqrt{\left(G E_{x}-\operatorname{Re}_{x}\right)^{2}+\left(G E_{y}+\operatorname{Re}_{y}\right)^{2}}
$$

where e stands for an endosome in the second channel. The smallest distance is the distance to the closest neighbor. $\triangle$ CRITICAL STEP Be careful not to include the beads as they will represent false-positive (colocalized) values.

50| Calculate how many of those spots have their intensity centers within a certain distance, depending on whether fusion or sorting/budding (A), or docking (B) should be measured:

\section{(A) Fusion or sorting/budding}

(i) The distance between the two intensity centers is $0-100 \mathrm{~nm}$.

\section{(B) Docking}

(i) The distance between the two intensity centers is $150-500 \mathrm{~nm}$.

$\Delta$ CRITICAL STEP If endosomes from cell types other than PC12 are used, other distance thresholds might be necessary. This can be checked by using endosomes that contain one cargo labeled with two fluorescent dyes. Bear in mind that double-labeled organelles still show some measurable distance between their green and red centers of intensity ${ }^{10}$. We also recommend testing the distance distribution obtained for multicolored fluorescent TetraSpeck beads (200 nm in diameter). We use this as our standard for double-labeled (fused) objects (Fig. 2).

$\Delta$ CRITICAL STEP Files for this data analysis routine (Steps 44-50), written in Matlab (The Mathworks Inc.), can be obtained from the authors on request.

51| Optionally, you can then distribute the intensity centers from one color randomly (e.g., by creating a 'mirror image').

52| Repeat Steps 49-50 using the 'mirror image'.

53| Subtract the values obtained from 'mirror images' from the real ones, to correct for random distribution of endosomes. ? TROUBLESHOOTING

Figure 2 | Imaging of multilabeled fluorescent beads. To determine the expected distance distribution for double-labeled (fused) organelles, we recommend calibrating the imaging setup by using multilabeled fluorescent TetraSpeck beads similar in size to the organelles of interest. (a) Images from 200-nm TetraSpeck beads in the green, red and blue channels, and an overlay image of the three channels together. Scale bar, $5 \mu \mathrm{m}$. (b) The distance distribution shows that, in our setup, only a minority of distances between the green and red centers of intensity are above $100 \mathrm{~nm}$ (dotted line). This confirms that this value is an accurate estimation of the fusion cutoff.
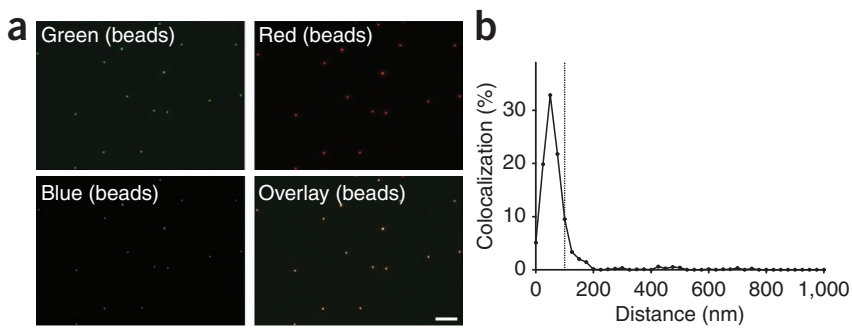
TIMING

Steps $1-8$, Preparation of rat brain cytosol: $4-5 \mathrm{~h}$ (day 1 )

Steps 9 and 10, Culture of PC12 cells: 30 min preparation + several days for cell culture (days 3-10)

Steps 11-30, Preparation of postnuclear supernatants: $3-5 \mathrm{~h}$ (day 11)

Steps 31-43, Preparation of docking/fusion or sorting/budding reactions: $2.5-10 \mathrm{~h}$ (day 12)

Steps 44-53 (data analysis): approximately $10 \mathrm{~min}$ for each imaged coverslip (day 13)

\section{? TROUBLESHOOTING}

Troubleshooting advice can be found in Table 1.

TABLE 1 | Troubleshooting table.

\begin{tabular}{|c|c|c|c|}
\hline Step & Problem & Possible reason & Solution \\
\hline 41 & $\begin{array}{l}\text { Unclear which } \\
\text { exposure times } \\
\text { should be used }\end{array}$ & $\begin{array}{l}\text { Bleedthrough between } \\
\text { different channels }\end{array}$ & $\begin{array}{l}\text { To check for bleedthrough, you should prepare PNS samples in which only } \\
\text { one of the fluorescent markers is endocytosed (only green or only red). } \\
\text { These samples should be treated (incubated) under identical conditions to } \\
\text { all other (multilabeled) samples and should be imaged in all color channels. } \\
\text { Exposure times should be adjusted so that no spots are visible in channels } \\
\text { other than the appropriate one }\end{array}$ \\
\hline
\end{tabular}

45 Unclear which threshold should be used

The colocalization in the control samples (ice or -ATP) is too high

No decrease in colocalization can be detected in the sorting/budding assay

Fusion rates are too low
Dim organelles or noisy background

Bleedthrough between different channels The density of organelles is too high

Fusion of the newly sorted organelles might be very high, thus obscuring the decrease in colocalization induced by sorting/budding

PNS fractions are poorly labeled, which may be due to (i) inefficient endocytosis (Step 22) or (ii) disruption of endosomes during homogenization (Step 28, particularly if fluid phase markers are used)
Start with a threshold equal to the mean image intensity (most spots will still be background); increase threshold by $\sim 5$ arbitrary units and continue until reaching the maximum intensity (essentially no spots are below the threshold). For each of the thresholds, compare the colocalization obtained for a control (e.g. -ATP, ice) versus a normally incubated sample. The threshold that provides the largest colocalization difference between the two samples should be used

Lower the exposure times in the appropriate channels (see TROUBLESHOOTING of Step 41)

Correct for random colocalization (Steps 51-53)

Use separate PNS fractions for both labels and check their fusion efficiency

Perform an immunostaining of the PNS for endosomal markers, to analyze how many of the endosomes are labeled. If labeling efficiency is too low, do the following: (i) increase amount of label (Step 22), (ii) make sure cell clumps are well dissociated (Step 9), (iii) shorten trypsin treatment (Step 14)

Perform ultracentrifugation of the labeled PNS and measure the amount of marker in the pellet (after detergent solubilization) and supernatant using fluorescence photometry or western blotting (using an antibody against the dye, e.g., Alexa 488, ref. 12). Typically, $70-90 \%$ of the label should be in the pellet. If the label is mostly in the supernatant, decrease the ball size to increase the clearance during homogenization (note that if the clearance is too high, cell cracking may become inefficient-see above) 
PROTOCOL

\section{ANTICIPATED RESULTS}

The fluorescence in vitro assays described here allow for studying docking and fusion, as well as sorting and budding of endosomes at the level of single organelles. Endosomes are labeled with different fluorescent endocytic tracers, which can be visualized using fluorescence microscopy. In our experimental setup, typically $10 \%$ of the endosomes were fluorescently labeled; this may vary somewhat from sample to sample, between $\sim 5 \%$ and $15 \%$. The distance between the labeled endosomes in the different fluorescence channels (e.g., green and red) serves as a measure of their interaction: green and red spots are closer together (in our setup, using PC12 endosomes, fused organelles have a distance of up to $\sim 100 \mathrm{~nm}$, with docked organelles between 150 and $500 \mathrm{~nm}$ ) when endosomes are fused (i.e., one organelle), compared with when they are docked (i.e., two closely apposed organelles). An accurate distance measurement between the spots of the two fluorescent channels can be achieved by using a control reaction prepared with multicolored fluorescent beads, which are used to align the images with precision. Typical images of endosomes and beads are shown in Figure $3 a$.

In the docking/fusion assay, two sets of single-labeled endosomes are mixed, leading to the formation of larger, doublelabeled organelles (yellow spots, arrowheads, only visible in the positive control; Fig. $\mathbf{3 b}$ ). To quantify the amount of docked and fused endosomes, for each green spot, we need to calculate the distance to its closest red neighbor. The results in Figure $3 \mathrm{c}$ show a typical histogram from one experiment. Both fusion (center-to-center distances within $100 \mathrm{~nm}$ ) and docking (within 150-500 nm) increase with incubation at $37^{\circ} \mathrm{C}$ (compared with the negative control kept on ice). Note that in fusion reactions one should not expect all organelles to fuse-typically $10-20 \%$ of the organelles fuse within the reaction time frame.

In the sorting/budding assay, one set of double-labeled endosomes is incubated under conditions that allow cargo sorting and budding, leading to the separation of the two labels. Thus, less double-labeled organelles (yellow spots, arrowheads) are visible in the positive control, compared with the negative control (Fig. 3d). The amount of double-labeled endosomes (within $100 \mathrm{~nm}$ ) decreases significantly on incubation at $37^{\circ} \mathrm{C}$ (Fig. 3e).

Figure 3 | Data obtained from docking/fusion and sorting/budding assays. (a) Images are taken in the two fluorescent channels that visualize the endosomes (e.g., green and red) and in one additional channel (blue) in which only multicolored fluorescent beads (used for image alignment, white circles) are visible. Overlaid image of green and red channels is also shown. Scale bar, $5 \mu \mathrm{m}$. (b) Images taken from the docking/fusion assay, in which endosomes are labeled with dextran-Alexa 488 (green) or dextran-Alexa 594 (red). The negative control was kept on ice (left) and the positive control was incubated at $37^{\circ} \mathrm{C}$ (right) in the presence of rat brain cytosol and an ATP-regenerating system. Many yellow spots appear in the positive control (arrowheads), representing docked or fused endosomes. As above, white circles indicate multicolored fluorescent beads. (c) Quantification of one docking/fusion experiment. Intensity centers for each spot in each channel are calculated and endosome images (green and red) are aligned by multicolored fluorescent beads. For each green endosome, the distance to its closest neighbor in

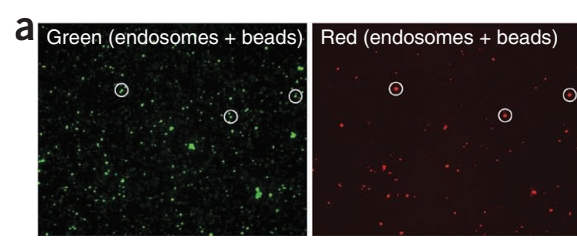

b

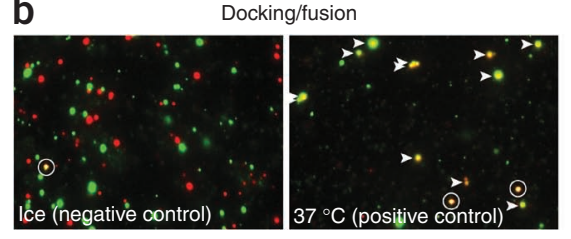

C

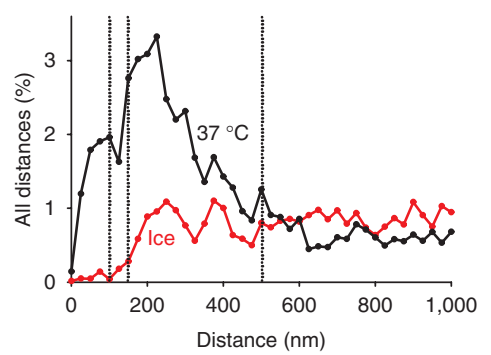

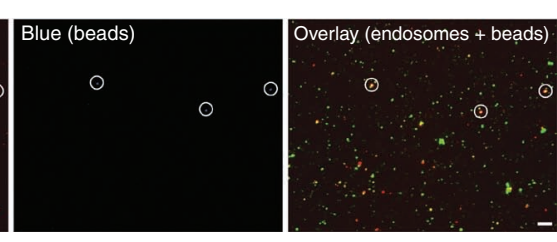

d

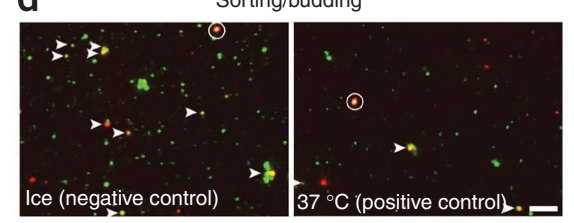

e

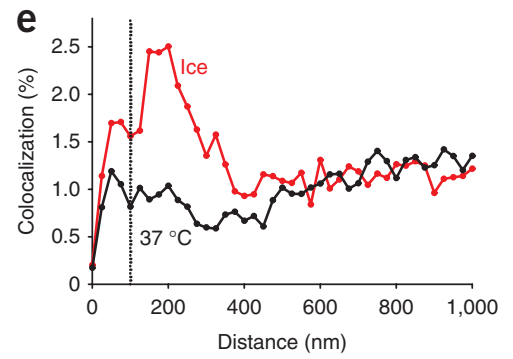

the red channel is then calculated and plotted in the histogram. Fusion (within 100-nm distance, before the first vertical dotted line) and docking (between 150- and 500-nm distance, delimited by the second and third vertical dotted lines) strongly increase with incubation at $37{ }^{\circ} \mathrm{C}$. Note that the histogram only shows distances up to $1,000 \mathrm{~nm}$, as larger distances represent only random positioning of the endosomes. Histograms represent data from one typical experiment (means of 14-16 images). (d) Images taken from the sorting/budding assay, in which endosomes are labeled with transferrin-Alexa 488 (green) or LDL-DiI (red). The negative control was kept on ice (left), and the positive control was incubated at $37^{\circ} \mathrm{C}$ (right) in the presence of rat brain cytosol and an ATP-regenerating system. Many double-labeled endosomes (yellow spots, arrowheads) are visible in the negative control, but are less frequent in the positive control. As above, white circles indicate the multicolored fluorescent beads. Scale bar, $5 \mu \mathrm{m}$. (e) Quantification was performed as in c. Endosomes that have their intensity center within $100 \mathrm{~nm}$ (vertical dotted line) represent double-labeled ones. Their number decreases significantly with incubation at $37{ }^{\circ} \mathrm{C}$. Histograms represent data from one typical experiment (means of 16-20 images). 
ACKNOWLEDGMENTS We thank D. Brandhorst, D. Zwilling, U. Geumann and I. Bethani for the contribution to the development of the method presented in this study. We thank M. Lengauer and F. Kötting for the help with the ball homogenizer. This work was supported by grants from the Deutsche Forschungsgemeinschaft (SFB 523 and SFB 803) to R.J. S.V.B. has been the recipient of a fellowship of the International Max Planck Research School 'Molecular Biology'.

AUTHOR CONTRIBUTIONS S.V.B., R.J. and S.O.R. conceived the experimental strategy; S.V.B. and S.O.R. designed the experiments; S.V.B. and S.0.R. conducted the experiments; S.V.B., R.J. and S.O.R. wrote the article.

COMPETING FINANCIAL INTERESTS The authors declare no competing financial interests.

Published online at http://www.natureprotocols.com/.

Reprints and permissions information is available online at http://npg.nature. com/reprintsandpermissions/

1. Gruenberg, J. \& Howell, K.E. Membrane traffic in endocytosis: insights from cell-free assays. Annu. Rev. Cell Biol. 5, 453-481 (1989).

2. Pagano, A., Crottet, P., Prescianotto-Baschong, C. \& Spiess, M. In vitro formation of recycling vesicles from endosomes requires adaptor protein-1/ clathrin and is regulated by rab4 and the connector rabaptin-5. Mol. Biol. Cell 15, 4990-5000 (2004).

3. Prekeris, R., Klumperman, J., Chen, Y.A. \& Scheller, R.H. Syntaxin 13 mediates cycling of plasma membrane proteins via tubulovesicular recycling endosomes. J. Cell Biol. 143, 957-971 (1998).

4. Desnos, C., Clift-0'Grady, L. \& Kelly, R.B. Biogenesis of synaptic vesicles in vitro. J. Cell Biol. 130, 1041-1049 (1995).

5. Clift-O'Grady, L. et al. Reconstitution of synaptic vesicle biogenesis from PC12 cell membranes. Methods 16, 150-159 (1998).

6. Lichtenstein, Y., Desnos, C., Faundez, V., Kelly, R.B. \& Clift-0'Grady, L. Vesiculation and sorting from PC12-derived endosomes in vitro. Proc. Natl Acad. Sci. USA 95, 11223-11228 (1998).
7. Lim, S.N. et al. Identification of discrete classes of endosome-derived small vesicles as a major cellular pool for recycling membrane proteins. Mol. Biol. Cell 12, 981-995 (2001).

8. de Wit, H. et al. Rab4 regulates formation of synaptic-like microvesicles from early endosomes in PC12 cells. Mol. Biol. Cell 12, 3703-3715 (2001).

9. Brandhorst, D. et al. Homotypic fusion of early endosomes: SNAREs do not determine fusion specificity. Proc. Natl Acad. Sci. USA 103, 2701-2706 (2006).

10. Geumann, U., Barysch, S.V., Hoopmann, P., Jahn, R. \& Rizzoli, S.0. SNARE function is not involved in early endosome docking. Mol. Biol. Cell 19, 5327-5337 (2008).

11. Bethani, I. et al. Endosomal fusion upon SNARE knockdown is maintained by residual SNARE activity and enhanced docking. Traffic 10, 1543-1559 (2009).

12. Barysch, S.V., Aggarwal, S., Jahn, R. \& Rizzoli, S.0. Sorting in early endosomes reveals connections to docking- and fusion-associated factors. Proc. Natl Acad. Sci. USA 106, 9697-9702 (2009).

13. Rizzoli, S.0. et al. Evidence for early endosome-like fusion of recently endocytosed synaptic vesicles. Traffic 7, 1163-1176 (2006).

14. Balch, W.E. \& Rothman, J.E. Characterization of protein transport between successive compartments of the Golgi apparatus: asymmetric properties of donor and acceptor activities in a cell-free system. Arch. Biochem. Biophys. 240, 413-425 (1985).

15. Lang, T. Imaging $\mathrm{Ca}^{2+}$-triggered exocytosis of single secretory granules on plasma membrane lawns from neuroendocrine cells. Methods Mol. Biol. 440, 51-59 (2008).

16. Bradford, M.M. A rapid and sensitive method for the quantitation of microgram quantities of protein utilizing the principle of protein-dye binding. Anal. Biochem. 72, 248-254 (1976).

17. Davis, J.M. Basic Cell Culture (Practical Approach Series) Edn. 2. (0xford University Press, 2002).

18. Russ, J.C. The Image Processing Handbook Edn. 5. (CRC Press, 2006). 